\title{
VASCULAR FLORA OF DRAINAGE DITCHES IN FOREST AREAS OF THE POLESIE NATIONAL PARK
}

\author{
Barbara Banach \\ Department of General Ecology, University of Life Sciences in Lublin \\ Akademicka 15, 20-950 Lublin, Poland \\ e-mail: barbara.banach@up.lublin.pl
}

Received: 29.09 .2008

\section{Abstract}

This paper presents the results of floristic studies conducted in the years 2003 - 2005 within the area of the Polesie National Park. The aim of the research was to compare the flora of initial succession stages in drainage ditches with the flora of neighbouring forest areas undergoing transformation as a result of drainage. Floristic lists were made in four designated study areas as well as in forest phytocoenoses. The results of field investigations were subjected to analysis with regard to the systematic affinity of the flora and proportions of plant life-forms as well as of historical-geographical groups and range groups.

In the species composition of the studied drainage ditches hemicryptophytes were the dominant group of plant higher life-forms, whereas in the neighbouring communities the flora was represented by both hemicryptophytes and megaphanerophytes.

In historical and geographical terms, the flora of drainage ditches and neighbouring communities represents definitely the group of spontaneophytes. Anthropogenic habitats, which developed within the drainage ditches, were colonised by native flora species characteristic for the Polesie National Park area.

Key words: species diversity, vascular flora, drainage ditches, Polesie National Park

\section{INTRODUCTION}

From the environmental point of view, the construction of the Wieprz-Krzna Canal had a very negative effect on the natural environment of the whole area situated within its impact zone. Water relations of this part of the area in question were drastically changed, the water discharge process intensified, which resulted in an absence of water stagnation ( $\mathrm{Ch} \mathrm{mi} \mathrm{e} \mathrm{le} \mathrm{w} \mathrm{s} \mathrm{k} \mathrm{i}$ and $\mathrm{Radwan}, 1993$ ), and the allochthonous waters, with different trophy and chemical composition, carried in the canal, contributed to changes in habi- tat conditions (W ilgat, 1991; M i chalc zy k and Turczyński 1998; Turczyński et al. 2000). Before the construction of the canal, temporary or permanent swamps accounted for $70 \%$ of the area which is currently occupied by the Polesie National Park, including its buffer zone. After carrying out land drainage works, a bare $15 \%$ of this valuable area resisted the anthropopressure process. The largest losses in flora abundance were noted in the Eęczna - Włodawa Lakeland, where the loss in natural resources of unique vegetation is estimated, depending on the type of plant community, at 60-95\% (Ch mie lew ski and $\mathrm{S}$ i elew i c z, 1994).

As early as 1959, Professor D o minik Fijałkowski drew attention to the fact that the Łęczna-Włodawa Lakeland area was still not sufficiently known in floristic and phytosociological terms. In connection with the construction of the canal, carried out at that time, and expected transformations which would take place after its completion, he postulated that it was necessary to undertake biological research in the near future (F i j a łk ow s ki, 1959). Since 1975 the topic of adverse changes taking place in the plant cover of the area impacted by the canal has been raised more and more frequently in scientific publications (Chmielewski, 1986). Since that time numerous studies have been undertaken and carried out, designed to get to know better the natural values of this area. Nevertheless, there are still numerous ecosystems and habitats which have not been fully investigated, and the knowledge of their plant cover is only superficial or approximate. Such special habitats include, among others, drainage ditches which are the object of investigation under the present study. 


\section{RESEARCH METHODS}

The studies on the flora of drainage ditches were carried out in the years 2003-2006, during which a detailed inventory covered four 100-metre-long sections of the drainage ditches intersecting both forest phytocoenoses and neighbouring communities. The study areas were designated with letters from A to D (Fig. 1). In each study area, representative plant patches were distinguished, within which lists of species were made. Inventories of herbaceous vegetation were conducted on an area of $25 \mathrm{~m}^{2}$, whereas of the flora of forest communities on an area of $400 \mathrm{~m}^{2}$. The ground cover of the respective plant patch by particular plant species was given in a 10-point scale. The degrees were calculated based on percentage density, where: $1^{\circ}$ corresponded to the percent cover of a particular species at a respective study area from 1 to $10 \%$, $2^{\circ}-11-20 \%, 3^{\circ}-21-30 \%, 4^{\circ}-31-40 \%, 5^{\circ}-41-50 \%$, $6^{\circ}-51-60 \%, 7^{\circ}-61-70 \%, 8^{\circ}-71-80 \%, 9^{\circ}-81-90 \%$ and $10^{\circ}$ which means that the density of a particular species is from 91 to $100 \%$. Species whose ground cover did not exceed $1 \%$ were designated in the lists by means of pluses (from + to +++ ), and species represented by a single specimen were designated by the letter " $r$ ".
Species requiring laboratory identification were collected and deposited in a herbarium. The prepared lists included the flora of both the ditch channel and its slopes. A total of 60 floristic lists were made in the drainage ditches and 8 in the neighbouring phytocoenoses. The data collected in field were used for determining the biodiversity of vascular flora of the studied ecosystems. In order to identify taxa, their affinity to historical-geographical groups and range groups was determined in accordance with Chmiel (1993b) as well as the following indicator values were calculated according to $\mathrm{Z}$ a r z y c ki et al. (2002):

- continentality index;

- life forms;

- number of stands;

- dynamic tendencies over the last decades.

The affinity of taxa to historical-geographical groups and range groups ( $\mathrm{Ch} \mathrm{miel,} 1993 \mathrm{~b})$ as well as the abovementioned indicator values $(\mathrm{Zarz} \mathrm{y} \mathrm{c} \mathrm{ki}$ et al. 2002) were used for further analysis of the flora. Percentage proportions of particular groups and indicator levels were calculated, whereas species being overlapping elements, representing several groups or levels of an indicator in question, were included both in representative groups and levels.

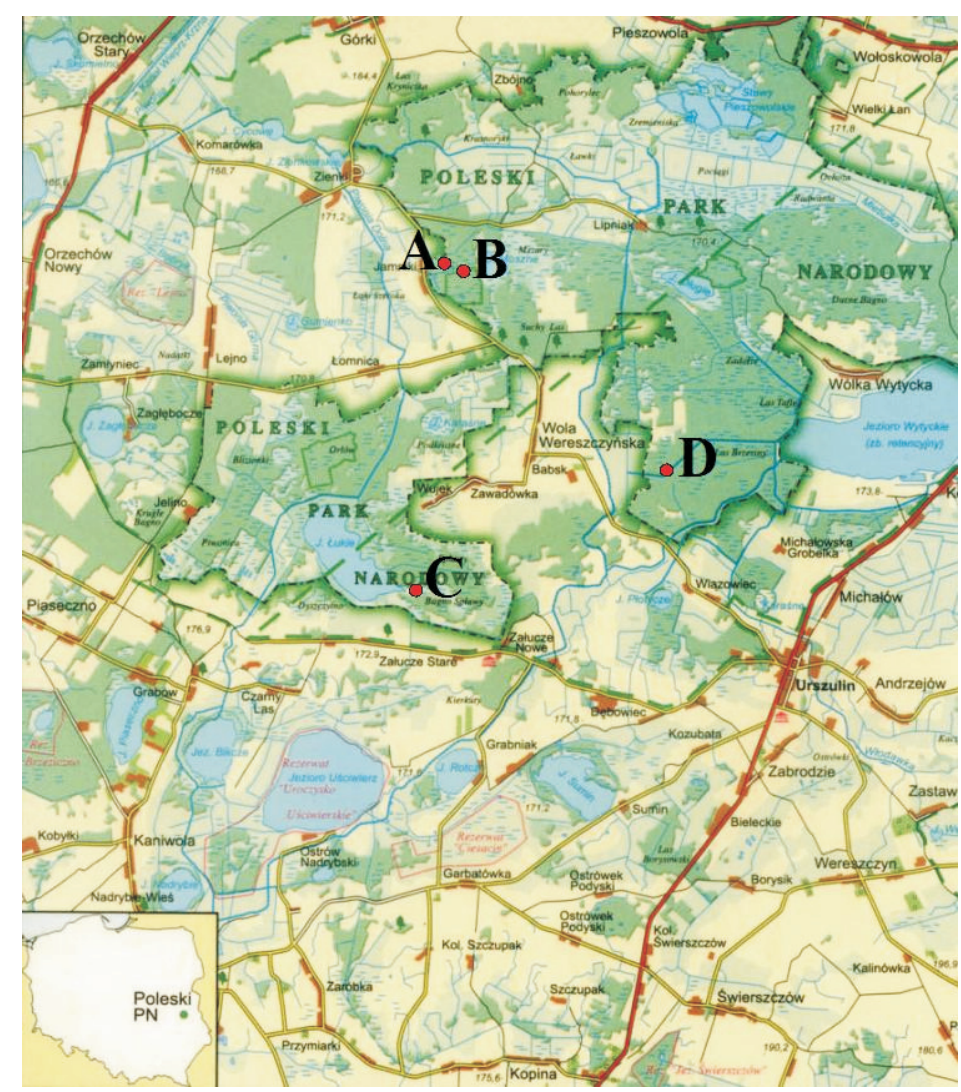

Fig. 1. Location of the study sites in the Polesie National Park area (Ka a a m u cki et al. 2001; changed). 
Table 1

Alphabetic list and ecological characterization of vascular plant species in the studied drainage ditches and neighbouring phytocoenoses.

\begin{tabular}{|c|c|c|c|c|c|c|c|}
\hline Latin name & Family & $\begin{array}{c}\text { Drainage } \\
\text { ditch }\end{array}$ & $\begin{array}{l}\text { Neighbour- } \\
\text { ing phyto- } \\
\text { coenoses }\end{array}$ & $\begin{array}{l}\text { Life } \\
\text { form }\end{array}$ & $\begin{array}{l}\text { Continen- } \\
\text { tality index }\end{array}$ & $\begin{array}{c}\text { Historical- } \\
\text { geographical } \\
\text { group }\end{array}$ & $\begin{array}{l}\text { Range } \\
\text { group }\end{array}$ \\
\hline Achillea millefolium $\mathrm{L}$. & Asteraceae & + & + & $\mathrm{H}$ & Ap & ES & 3 \\
\hline Aegopodium podagraria $\mathrm{L}$. & Apiaceae & + & & $\mathrm{G}, \mathrm{H}$ & $\mathrm{Sp}$ & ES & 3 \\
\hline Agrostis capillaris L. & Poaceae & + & + & $\mathrm{H}$ & Ap & ES & 3 \\
\hline Agrostis gigantea Roth & Poaceae & + & + & $\mathrm{H}$ & Ap & ES & 3 \\
\hline Agrostis stolonifera L. & Poaceae & + & & $\mathrm{H}$ & Ap & ES & 3 \\
\hline Alisma plantago-aquatica $\mathrm{L}$. & Alismataceae & + & & Hy & Ap & KOSM & 3 \\
\hline Alnus glutinosa (L.) Gaertn. & Betulaceae & + & + & M & $\mathrm{Sp}$ & ES & 3 \\
\hline Anemone nemorosa $\mathrm{L}$. & Ranunculaceae & + & & $\mathrm{G}, \mathrm{H}$ & $\mathrm{Sp}$ & $\mathrm{E}$ & 3 \\
\hline Aquilegia vulgaris L. & Ranunculaceae & & + & $\mathrm{H}$ & $\mathrm{Sp}$ & $\mathrm{E}$ & 3 \\
\hline Betula pendula Roth & Betulaceae & + & + & M & Ap & ES & $3 / 4$ \\
\hline Betula pubescens Ehrh. & Betulaceae & + & + & M & $\mathrm{Sp}$ & ES & 3 \\
\hline Bidens tripartita $\mathrm{L}$ & Asteraceae & + & & $\mathrm{T}$ & Ap & M-ES & 3 \\
\hline $\begin{array}{l}\text { Calamagrostis canescens (Weber) } \\
\text { Roth }\end{array}$ & Poaceae & + & & $\mathrm{H}$ & $\mathrm{Sp}$ & ES & 3 \\
\hline Calla palustris $\mathrm{L}$ & Araceae & + & + & Hy, G & Sp & $\mathrm{CB}$ & 3 \\
\hline Calluna vulgaris (L.) Hull & Ericaceae & & + & $\mathrm{Ch}$ & $\mathrm{Sp}$ & sOZ-E & 3 \\
\hline Caltha palustris $\mathrm{L}$. & Ranunculaceae & + & & $\mathrm{H}$ & $\mathrm{Sp}$ & $\mathrm{CB}$ & 3 \\
\hline Calystegia sepium (L.) R.Br. & Convolvulaceae & + & + & $\begin{array}{c}\mathrm{G}, \mathrm{H}, \\
\mathrm{li}\end{array}$ & $\mathrm{Sp}$ & KOSM & 3 \\
\hline Carex acutiformis Ehrh. & Cyperaceae & + & & $\mathrm{G}, \mathrm{Hy}$ & $\mathrm{Sp}$ & ES & 3 \\
\hline Carex canescens $\mathrm{L}$. & Cyperaceae & + & & $\mathrm{H}$ & . & . & 3 \\
\hline Carex elata All. & Cyperaceae & + & + & $\mathrm{H}, \mathrm{Hy}$ & $\mathrm{Sp}$ & $\mathrm{sOZ}$ & 3 \\
\hline Carex elongata $\mathrm{L}$. & Cyperaceae & + & & $\mathrm{H}$ & $\mathrm{Sp}$ & ES & 3 \\
\hline Carex gracilis Curtis & Cyperaceae & + & & $\mathrm{G}, \mathrm{Hy}$ & . & . & 3 \\
\hline Carex hirta $\mathrm{L}$ & Cyperaceae & + & + & G & Ap & M-E & 3 \\
\hline Carex lasiocarpa Ehrh. & Cyperaceae & + & & $\mathrm{H}, \mathrm{Hy}$ & $\mathrm{Sp}$ & $\mathrm{CB}$ & 4 \\
\hline Carex nigra Reichard & Cyperaceae & + & & $\mathrm{G}$ & $\mathrm{Sp}$ & ES-AM & 3 \\
\hline Carex ovalis Gooden. & Cyperaceae & & + & $\mathrm{H}$ & Ap & ES & 3 \\
\hline Carex pairae F. W. Schultz & Cyperaceae & + & & $\mathrm{H}$ & . & . & 3 \\
\hline Carex panicea $\mathrm{L}$ & Cyperaceae & + & & $\mathrm{G}, \mathrm{H}$ & $\mathrm{Sp}$ & ES-AM & 3 \\
\hline Carex paniculata $\mathrm{L}$. & Cyperaceae & + & + & $\mathrm{H}$ & . & . & 3 \\
\hline Carex pseudocyperus L. & Cyperaceae & + & + & $\mathrm{H}, \mathrm{Hy}$ & $\mathrm{Sp}$ & KOSM & 3 \\
\hline Carex riparia Curtis & Cyperaceae & + & & $\mathrm{H}, \mathrm{Hy}$ & $\mathrm{Sp}$ & KOSM & 3 \\
\hline Carex rostrata Stokes & Cyperaceae & + & & $\mathrm{H}, \mathrm{Hy}$ & $\mathrm{Sp}$ & $\mathrm{CB}$ & 3 \\
\hline Cicuta virosa $\mathrm{L}$ & Apiaceae & + & & $\mathrm{H}$ & $\mathrm{Sp}$ & ES & 3 \\
\hline Cirsium palustre (L.) Scop. & Asteraceae & + & & $\mathrm{H}$ & $\mathrm{Sp}$ & M-ES & 3 \\
\hline Comarum palustre $\mathrm{L}$. & Rosaceae & + & + & $\mathrm{Ch}$ & . & . & 3 \\
\hline Consolida regalis Gray & Ranunculaceae & & + & $\mathrm{T}$ & Arch & ES-P & 3 \\
\hline Crataegus monogyna Jacq. & Rosaceae & & + & $\mathrm{N}$ & Ap & M-E-IT & 3 \\
\hline Deschampsia caespitosa (L.) P.Beauv. & Poaceae & + & + & $\mathrm{H}$ & $\mathrm{Sp}$ & KOSM & 3 \\
\hline Dryopteris filix-mas (L.) Schott & Dryopteridaceae & + & & $\mathrm{H}$ & $\mathrm{Sp}$ & KOSM & 3 \\
\hline Elymus repens (L.) Gould & Poaceae & + & & G & Ap & M-ES & 3 \\
\hline Epilobium palustre $\mathrm{L}$. & Onagraceae & + & & $\mathrm{H}$ & $\mathrm{Sp}$ & $\mathrm{CB}$ & 3 \\
\hline Equisetum fluviatile L. & Equisetaceae & + & + & Hy, G & $\mathrm{Sp}$ & $\mathrm{CB}$ & 3 \\
\hline Equisetum palustre $\mathrm{L}$. & Equisetaceae & + & & G & $\mathrm{Sp}$ & $\mathrm{M}-\mathrm{CB}$ & 3 \\
\hline Equisetum sylvaticum $\mathrm{L}$. & Equisetaceae & + & + & G & Ap & $\mathrm{CB}$ & 3 \\
\hline Eriophorum vaginatum $\mathrm{L}$. & Cyperaceae & & + & $\mathrm{H}$ & $\mathrm{Sp}$ & $\mathrm{CB}$ & 3 \\
\hline Filipendula ulmaria (L.) Maxim. & Rosaceae & + & & $\mathrm{H}$ & $\mathrm{Sp}$ & ES & 3 \\
\hline Fragaria vesca $\mathrm{L}$ & Rosaceae & & + & $\mathrm{H}$ & $\mathrm{Sp}$ & $\mathrm{CB}$ & 3 \\
\hline
\end{tabular}


cd. Table 1

\begin{tabular}{|c|c|c|c|c|c|c|c|}
\hline Latin name & Family & $\begin{array}{c}\text { Drainage } \\
\text { ditch }\end{array}$ & $\begin{array}{c}\text { Neighbour- } \\
\text { ing phyto- } \\
\text { coenoses }\end{array}$ & $\begin{array}{l}\text { Life } \\
\text { form }\end{array}$ & $\begin{array}{c}\text { Continen- } \\
\text { tality index }\end{array}$ & $\begin{array}{c}\text { Historical- } \\
\text { geographical } \\
\text { group }\end{array}$ & $\begin{array}{l}\text { Range } \\
\text { group }\end{array}$ \\
\hline $\begin{array}{l}\text { Frangula alnus Mill. } \\
\text { Galeobdolon luteum Huds. }\end{array}$ & $\begin{array}{l}\text { Rhamnaceae } \\
\text { Lamiaceae }\end{array}$ & + & $\begin{array}{l}+ \\
+\end{array}$ & $\begin{array}{l}\mathrm{N} \\
\mathrm{Ch}\end{array}$ & $\mathrm{Sp}$ & ES & $\begin{array}{l}3 \\
3\end{array}$ \\
\hline Galeopsis speciosa Mill. & Lamiaceae & + & & $\mathrm{T}$ & Ap & ES & 3 \\
\hline Galium aparine $\mathrm{L}$. & Rubiaceae & + & + & $\mathrm{T}, \mathrm{H}$ & Ap & ES & 3 \\
\hline Galium palustre L. & Rubiaceae & + & & $\mathrm{H}$ & $\mathrm{Sp}$ & M-ES-AM & 3 \\
\hline Galium uliginosum $\mathrm{L}$. & Rubiaceae & + & & $\mathrm{H}$ & $\mathrm{Sp}$ & ES & 3 \\
\hline Genista pilosa $\mathrm{L}$. & Fabaceae & + & + & $\mathrm{Ch}$ & . & . & 2 \\
\hline Geranium robertianum $\mathrm{L}$. & Geraniaceae & + & + & $\mathrm{H}, \mathrm{T}$ & $\mathrm{Sp}$ & M-CB & 3 \\
\hline Geum rivale $\mathrm{L}$. & Rosaceae & + & + & $\mathrm{H}$ & $\mathrm{Sp}$ & M-E-AM & 3 \\
\hline Geum urbanum L. & Rosaceae & & + & $\mathrm{H}$ & Ap & M-E-IT & 3 \\
\hline Glyceria fluitans (L.) R.Br. & Poaceae & + & + & Нy & Ap & M-E-AM & 3 \\
\hline Hieracium murorum L. & Asteraceae & + & + & $\mathrm{H}$ & $\mathrm{Sp}$ & E & 3 \\
\hline Hieracium pilosella $\mathrm{L}$. & Asteraceae & & + & $\mathrm{H}$ & Ap & $\mathrm{E}$ & 3 \\
\hline Holcus lanatus L. & Poaceae & + & + & $\mathrm{H}$ & Ap & M-E & 3 \\
\hline Hottonia palustris L. & Primulaceae & + & & Hy & $\mathrm{Sp}$ & $\mathrm{E}$ & 3 \\
\hline Hydrocharis morsus-ranae L. & Hydrocharitaceae & + & & Hy & $\mathrm{Sp}$ & ES & 3 \\
\hline Hypericum perforatum $\mathrm{L}$. & Hypericaceae & + & + & $\mathrm{H}$ & Ap & ES & 3 \\
\hline Iris pseudacorus $\mathrm{L}$. & Iridaceae & + & & G, Hy & $\mathrm{Sp}$ & M-ES & 3 \\
\hline Juncus effusus L. & Juncaceae & + & & $\mathrm{H}$ & Ap & KOSM & 3 \\
\hline Lathyrus pratensis $\mathrm{L}$. & Fabaceae & + & & $\mathrm{H}$ & Ap & KOSM & 3 \\
\hline Lembotropis nigricans (L.) Grisb. & Fabaceae & & + & $\mathrm{C}, \mathrm{N}$ & $\mathrm{Sp}$ & E & 3 \\
\hline Leontodon autumnalis $\mathrm{L}$. & Asteraceae & & + & $\mathrm{H}$ & Ap & ES & 3 \\
\hline Lemna minor $\mathrm{L}$. & Lemnaceae & + & & Hy & $\mathrm{Sp}$ & KOSM & 3 \\
\hline Lemna trisulca $\mathrm{L}$. & Lemnaceae & + & & Hy & $\mathrm{Sp}$ & KOSM & 3 \\
\hline Lychnis flos-cuculi L. & Caryophyllaceae & + & & $\mathrm{H}$ & $\mathrm{Sp}$ & ES & 3 \\
\hline Lycopus europaeus L. & Lamiaceae & + & + & $\mathrm{H}, \mathrm{Hy}$ & $\mathrm{Sp}$ & M-ES & 3 \\
\hline Lysimachia nummularia $\mathrm{L}$. & Primulaceae & + & & $\mathrm{C}$ & $\mathrm{Sp}$ & E & 3 \\
\hline Lysimachia thyrsiflora L. & Primulaceae & + & + & H, Hy & $\mathrm{Sp}$ & $\mathrm{CB}$ & 3 \\
\hline Lysimachia vulgaris $\mathrm{L}$. & Primulaceae & + & + & $\mathrm{H}$ & $\mathrm{Sp}$ & ES & 3 \\
\hline Lythrum salicaria $\mathrm{L}$. & Lythraceae & + & & $\mathrm{H}$ & $\mathrm{Sp}$ & KOSM & 3 \\
\hline $\begin{array}{l}\text { Maianthemum bifolium (L.) } \\
\text { F.W.Schmidt }\end{array}$ & Convallariaceae & + & & G & $\mathrm{Sp}$ & ES & 3 \\
\hline Melandrium album (Mill.) Garcke & Caryophyllaceae & + & & $\mathrm{T}, \mathrm{H}$ & Ap & ES & 3 \\
\hline Mentha aquatica $\mathrm{L}$. & Lamiaceae & + & & H, Hy & $\mathrm{Sp}$ & KOSM & 3 \\
\hline Menyanthes trifoliata $\mathrm{L}$. & Menyanthaceae & & + & $\mathrm{G}, \mathrm{Hy}$ & $\mathrm{Sp}$ & $\mathrm{CB}$ & 3 \\
\hline Milium effusum $\mathrm{L}$. & Poaceae & & + & $\mathrm{H}$ & $\mathrm{Sp}$ & $\mathrm{CB}$ & 3 \\
\hline Molinia caerulea (L.) Moench & Poaceae & + & + & $\mathrm{H}$ & $\mathrm{Sp}$ & $\mathrm{E}$ & 3 \\
\hline Mycelis muralis (L.) Dumort. & Asteraceae & & + & $\mathrm{H}$ & $\mathrm{Sp}$ & sOZ & 3 \\
\hline $\begin{array}{l}\text { Myosotis palustris (L.) L.emend. } \\
\text { Rchb. }\end{array}$ & Boraginaceae & + & & $\mathrm{H}$ & . & . & 3 \\
\hline Myosoton aquaticum (L.) Moench & Caryophyllaceae & + & & $\mathrm{G}, \mathrm{H}$ & Ap & ES & 3 \\
\hline Oenanthe aquatica (L.) Poir. & Apiaceae & + & & H, Hy & Ap & ES & 3 \\
\hline Oxalis acetosella $\mathrm{L}$. & Oxalidaceae & + & + & $\mathrm{G}, \mathrm{H}$ & $\mathrm{Sp}$ & ES & 3 \\
\hline Oxalis stricta $\mathrm{L}$ & Oxalidaceae & + & & G & . & . & 3 \\
\hline Peucedanum palustre (L.) Moench & Apiaceae & + & & $\mathrm{H}$ & . & . & 3 \\
\hline $\begin{array}{l}\text { Phragmites australis (Cav.) Trin. ex } \\
\text { Steud. }\end{array}$ & Poaceae & + & + & G, Hy & $\mathrm{Sp}$ & KOSM & 3 \\
\hline Pimpinella saxifraga $\mathrm{L}$. & Apiaceae & + & & $\mathrm{H}$ & Ap & ES & 3 \\
\hline Pinus sylvestris L. & Pinaceae & & + & M & Ap & ES & 3 \\
\hline Plantago lanceolata $\mathrm{L}$. & Plantaginaceae & + & + & $\mathrm{H}$ & Ap & M-ES-IT & 3 \\
\hline Poa nemoralis L. & Poaceae & + & & $\mathrm{H}$ & $\mathrm{Sp}$ & $\mathrm{CB}$ & 3 \\
\hline Poa palustris L. & Poaceae & + & & $\mathrm{H}$ & $\mathrm{Sp}$ & $\mathrm{CB}$ & 3 \\
\hline
\end{tabular}




\begin{tabular}{|c|c|c|c|c|c|c|c|}
\hline Latin name & Family & $\begin{array}{l}\text { Drainage } \\
\text { ditch }\end{array}$ & $\begin{array}{c}\text { Neighbour- } \\
\text { ing phyto- } \\
\text { coenoses }\end{array}$ & $\begin{array}{l}\text { Life } \\
\text { form }\end{array}$ & $\begin{array}{l}\text { Continen- } \\
\text { tality index }\end{array}$ & $\begin{array}{c}\text { Historical- } \\
\text { geographical } \\
\text { group }\end{array}$ & $\begin{array}{l}\text { Range } \\
\text { group }\end{array}$ \\
\hline Poa pratensis L. & Poaceae & & + & $\mathrm{H}$ & Ap & $\mathrm{CB}$ & 3 \\
\hline Polygonatum multiflorum (L.) All. & Convallariaceae & & + & $\mathrm{G}$ & $\mathrm{Sp}$ & $\mathrm{ES}$ & 3 \\
\hline Populus tremula $\mathrm{L}$. & Salicaceae & + & + & M & Ap & ES & 3 \\
\hline Potentilla anserina $\mathrm{L}$. & Rosaceae & + & & $\mathrm{H}$ & Ap & KOSM & 3 \\
\hline Potentilla erecta (L.) Raeusch. & Rosaceae & + & & $\mathrm{H}$ & $\mathrm{Sp}$ & M-ES & 3 \\
\hline Potentilla reptans $\mathrm{L}$. & Rosaceae & + & & $\mathrm{H}$ & Ap & M-E-IT & 3 \\
\hline Pteridium aquilinum (L.) Kuhn & Dennstaedtiaceae & + & + & $\mathrm{G}$ & $\mathrm{Sp}$ & KOSM & 3 \\
\hline Quercus robur $\mathrm{L}$. & Fagaceae & + & + & M & $\mathrm{Sp}$ & $\mathrm{E}$ & 3 \\
\hline Ranunculus acris L. s. str. & Ranunculaceae & + & & $\mathrm{H}$ & Ap & $\mathrm{ES}$ & 3 \\
\hline Ranunculus flammula L. & Ranunculaceae & + & & $\mathrm{H}$ & Ap & $\mathrm{ES}$ & 3 \\
\hline Ranunculus lingua L. & Ranunculaceae & + & + & H, Hy & $\mathrm{Sp}$ & $\mathrm{ES}$ & 3 \\
\hline Rorippa amphibia (L.) Besser & Brassicaceae & + & & H, Hy & $\mathrm{Sp}$ & ES & 3 \\
\hline Rubus caesius $\mathrm{L}$. & Rosaceae & + & + & $\mathrm{N}, \mathrm{li}$ & Ap & ES-IT & 3 \\
\hline Rubus idaeus L. & Rosaceae & + & & $\mathrm{N}, \mathrm{li}$ & $\mathrm{Sp}$ & CB & 3 \\
\hline Rumex hydrolapathum Huds. & Polygonaceae & + & & H, Hy & $\mathrm{Sp}$ & $\mathrm{E}$ & 3 \\
\hline Salix aurita $\mathrm{L}$ & Salicaceae & + & & $\mathrm{N}$ & $\mathrm{Sp}$ & $\mathrm{E}$ & 3 \\
\hline Salix cinerea $\mathrm{L}$. & Salicaceae & + & + & $\mathrm{N}$ & $\mathrm{Sp}$ & $\mathrm{ES}$ & 3 \\
\hline Scirpus sylvaticus L. & Cyperaceae & + & & $\mathrm{G}$ & $\mathrm{Sp}$ & $\mathrm{ES}$ & 3 \\
\hline Scutellaria galericulata $\mathrm{L}$. & Lamiaceae & + & & $\mathrm{H}$ & $\mathrm{Sp}$ & CB & 3 \\
\hline Solanum dulcamara L. & Solanaceae & + & & $\mathrm{N}, \mathrm{li}$ & $\mathrm{Sp}$ & M-ES-IT & 3 \\
\hline Sorbus aucuparia L. emend. Hedl. & Rosaceae & + & + & $\mathrm{M}, \mathrm{N}$ & $\mathrm{Sp}$ & ES & 3 \\
\hline Stachys palustris L. & Lamiaceae & + & & G & $\mathrm{Sp}$ & $\mathrm{CB}$ & 3 \\
\hline Stellaria palustris Retz. & Caryophyllaceae & + & & $\mathrm{H}$ & $\mathrm{Sp}$ & $\mathrm{M}-\mathrm{CB}$ & 3 \\
\hline Stratiotes aloides L. & Hydrocharitaceae & & & Hy & $\mathrm{Sp}$ & ES & 3 \\
\hline Thelypteris palustris Schott & Thelypteridaceae & + & + & $\mathrm{G}$ & $\mathrm{Sp}$ & KOSM & 3 \\
\hline Tilia cordata Mill. & Tiliaceae & & + & M & $\cdot$ & . & 3 \\
\hline Trientalis europaea L. & Primulaceae & + & + & $\mathrm{G}$ & $\mathrm{Sp}$ & $\mathrm{CB}$ & 3 \\
\hline Triglochin palustre L. & Juncaginaceae & + & & $\mathrm{H}$ & $\mathrm{Sp}$ & KOSM & 3 \\
\hline Tussilago farfara $\mathrm{L}$. & Asteraceae & + & & G & Ap & ES & 3 \\
\hline Typha angustifolia L. & Typhaceae & + & & H, Hy & $\mathrm{Sp}$ & ES-AM & 3 \\
\hline Typha latifolia $\mathrm{L}$. & Typhaceae & + & & H, Hy & $\mathrm{Sp}$ & KOSM & 3 \\
\hline Urtica dioica $\mathrm{L}$ & Urticaceae & + & + & $\mathrm{H}$ & Ap & $\mathrm{M}-\mathrm{CB}$ & 3 \\
\hline Utricularia vulgaris L. & Lentibulariaceae & + & & Hy & $\mathrm{Sp}$ & ES & 3 \\
\hline Vaccinium myrtillus L. & Ericaceae & + & + & $\mathrm{Ch}$ & $\mathrm{Sp}$ & $\mathrm{ES}$ & 3 \\
\hline Vaccinium uliginosum L. & Ericaceae & & + & $\mathrm{Ch}$ & $\mathrm{Sp}$ & $\mathrm{CB}$ & 3 \\
\hline Vaccinium vitis-idaea L. & Ericaceae & & + & $\mathrm{Ch}$ & $\mathrm{Sp}$ & $\mathrm{CB}$ & 3 \\
\hline Veronica chamaedrys L. & Scrophulariaceae & + & + & $\mathrm{C}$ & Ap & $\mathrm{E}$ & 3 \\
\hline Veronica scutellata L. & Scrophulariaceae & + & & $\mathrm{H}$ & $\mathrm{Sp}$ & $\mathrm{CB}$ & 3 \\
\hline Vicia angustifolia L. & Fabaceae & + & + & $\mathrm{T}, \mathrm{H}$ & $\cdot$ & . & 3 \\
\hline Vicia tetrasperma (L.) Schreb. & Fabaceae & & + & $\mathrm{T}$ & Arch & M-P & 3 \\
\hline Viola palustris L. & Violaceae & + & + & $\mathrm{H}$ & $\mathrm{Sp}$ & E-AM & 3 \\
\hline $\begin{array}{l}\text { Viola reichenbachiana Jord. ex } \\
\text { Boreau }\end{array}$ & Violaceae & + & + & $\mathrm{H}$ & $\mathrm{Sp}$ & $\mathrm{E}$ & 3 \\
\hline
\end{tabular}

\section{Explanations:}

Life form (Zarzycki et al., 2002); M - megaphanerophytes, trees generally growing to a height of over 5 m, $\mathrm{N}-$ nanophanerophytes, shrubs and small trees, from $0.5 \mathrm{up}$ to $5 \mathrm{~m}$ in height, $\mathrm{Ch}$ - woody chamaephytes (buds $>25 \mathrm{~cm}$ above ground), $\mathrm{C}-$ herbaceous chamaephytes (buds $<25 \mathrm{~cm}$ above ground), $\mathrm{H}$ - hemicryptophytes (buds at ground level), $\mathrm{G}$ - geophytes (buds in soil), $\mathrm{T}-$ therophytes (annuals), Hy - hydrophytes and helophytes (buds In water), li - lianas (plants rooted in soil, requiring supports), pp - semiparasites,

Historical-geographical group (Chmiel, 1993 b); Sp - spontaneophytes, Ap - apophytes, Arch - archeophytes, Ken - kenophytes, D - diaphytes, $\bullet$ species of undetermined affinity,

Range group (Chmiel, 1993 b) CB - circumboreal, ES - Euro-Siberian, E - Central European, sOZ - sub-Atlantic, P - Pontian-Pannonian, ZAS - Central Asiatic, M - Mediterranean, IT - Irano-Turanian, AM - American, KOSM - cosmopolitan,

Continentality index (Zarzycki et al., 2002); 2 - sub-Atlantic species, found mainly in the western part of Poland, 3 - species neutral to continentality, 4 - subcontinental species, found mainly in the eastern part of Poland, $\bullet$ species of undetermined affinity. 
Table 2

Proportions of main taxonomic groups in the flora of the studied drainage ditches and neighbouring phytocoenoses.

\begin{tabular}{|c|c|c|c|c|c|c|c|c|c|c|c|c|}
\hline \multirow{3}{*}{ Taxa } & \multicolumn{4}{|c|}{ Families } & \multicolumn{4}{|c|}{ Genera } & \multicolumn{4}{|c|}{ Species } \\
\hline & \multicolumn{2}{|c|}{ Study area } & \multicolumn{2}{|c|}{$\begin{array}{l}\text { Neighbouring } \\
\text { phytocoenosis }\end{array}$} & \multicolumn{2}{|c|}{ Study area } & \multicolumn{2}{|c|}{$\begin{array}{l}\text { Neighbouring } \\
\text { phytocoenosis }\end{array}$} & \multicolumn{2}{|c|}{ Study area } & \multicolumn{2}{|c|}{$\begin{array}{l}\text { Neighbouring } \\
\text { phytocoenosis }\end{array}$} \\
\hline & number & $\%$ & number & $\%$ & number & $\%$ & number & $\%$ & number & $\%$ & number & $\%$ \\
\hline \multicolumn{13}{|c|}{ Phylum: Pteridophyta } \\
\hline $\begin{array}{l}\text { Class: } \\
\text { Sphenopsida }\end{array}$ & 1 & 2.3 & 1 & 3.4 & 1 & 1.2 & 1 & 1.8 & 3 & 2.6 & 2 & 2.9 \\
\hline $\begin{array}{l}\text { Class: } \\
\text { Filocopsida }\end{array}$ & 3 & 6.8 & 2 & 6.8 & 3 & 3.7 & 2 & 3.6 & 3 & 2.6 & 2 & 2.9 \\
\hline \multicolumn{13}{|c|}{ Phylum: Spermatophyta } \\
\hline $\begin{array}{l}\text { Subphylum: } \\
\text { Pinophytina }\end{array}$ & 0 & 0 & 1 & 3.4 & 0 & 0 & 1 & 1.8 & 0 & 0 & 1 & 1.4 \\
\hline \multicolumn{13}{|c|}{ Subphylum: Magnoliophytina } \\
\hline $\begin{array}{l}\text { Class: } \\
\text { Magnoliopsida }\end{array}$ & 30 & 68.2 & 23 & 79.3 & 57 & 70.4 & 41 & 74.6 & 71 & 61.7 & 49 & 71.1 \\
\hline Class: Liliopsida & 10 & 22.7 & 2 & 6.8 & 20 & 24.7 & 10 & 18.2 & 38 & 33.1 & 15 & 21.7 \\
\hline Total & 44 & 100 & 29 & 100 & 81 & 100 & 55 & 100 & 115 & 100 & 69 & 100 \\
\hline
\end{tabular}

Table 3

Proportions of life forms in the flora of the studied drainage ditches and neighbouring phytocoenoses.

\begin{tabular}{l|c|c|c|c}
\hline \multirow{2}{*}{ Life forms } & \multicolumn{2}{|c|}{ Study area } & \multicolumn{2}{c}{ Neighbouring phytocoenosis } \\
\cline { 2 - 5 } & number of species & $\begin{array}{c}\text { proportion } \\
\text { in \% }\end{array}$ & number of species & $\begin{array}{c}\text { proportion } \\
\text { in \% }\end{array}$ \\
\hline M & 6 & 5.2 & 8 & 11.6 \\
N & 7 & 6.1 & 6 & 8.7 \\
Ch & 2 & 1.7 & 7 & 10.1 \\
C & 2 & 1.7 & 2 & 2.9 \\
H & 70 & 60.9 & 35 & 50.7 \\
G & 25 & 21.7 & 12 & 17.4 \\
T & 6 & 5.2 & 5 & 7.2 \\
Hy & 28 & 24.3 & 10 & 14.5 \\
li & 4 & 3.5 & 2 & 2.9 \\
\hline
\end{tabular}

$\mathbf{M}$ - megaphanerophytes, trees generally growing to a height of over $5 \mathrm{~m}, \mathbf{N}$ - nanophanerophytes, shrubs and small trees, from 0.5 up to $5 \mathrm{~m}$ in height, $\mathbf{C h}$ - woody chamaephytes (buds $>25 \mathrm{~cm}$ above ground), $\mathbf{C}-$ herbaceous chamaephytes (buds $<25 \mathrm{~cm}$ above ground), $\mathbf{H}$ - hemicryptophytes (buds at ground level), $\mathbf{G}$ - geophytes (buds in soil), $\mathbf{T}$ - therophytes (annuals), Hy - hydrophytes and helophytes (buds In water), li - lianas (plants rooted in soil, requiring supports).

Table 4

Proportions of historical and geographical groups in the flora of drainage ditches and neighbouring phytocoenoses.

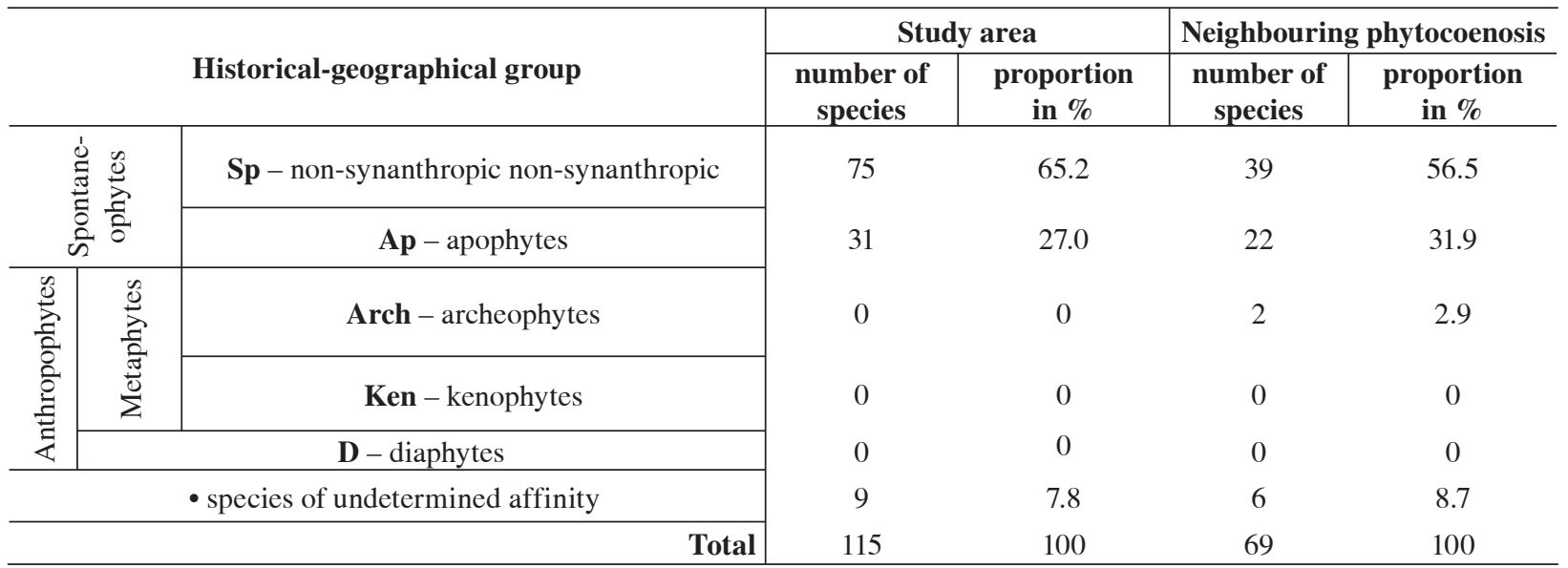


Table 5

Geographical elements in the flora of drainage ditches and neighbouring phytocoenoses.

\begin{tabular}{l|c|c|c|c}
\hline \multirow{2}{*}{\multicolumn{1}{c}{ Geographical (range) element }} & \multicolumn{2}{c|}{ Study area } & \multicolumn{2}{c}{ Neighbouring phytocoenosis } \\
\cline { 2 - 5 } & $\begin{array}{c}\text { number of } \\
\text { species }\end{array}$ & $\begin{array}{c}\text { proportion } \\
\text { in \% }\end{array}$ & $\begin{array}{c}\text { number of } \\
\text { species }\end{array}$ & $\begin{array}{c}\text { proportion } \\
\text { in \% }\end{array}$ \\
\hline CB - circumboreal & 15 & 13.0 & 12 & 17.4 \\
ES - Euro-Siberian & 39 & 33.9 & 20 & 29.0 \\
E - Central European & 10 & 8.7 & 8 & 11.6 \\
sOZ - sub-Atlantic & 1 & 0.9 & 2 & 2.9 \\
overlapping elements & 23 & 20.0 & 15 & 21.7 \\
KOSM - cosmopolitan & 18 & 15.7 & 6 & 8.7 \\
$\bullet$ - species of undetermined affinity & 9 & 7.8 & 6 & 8.7 \\
\hline
\end{tabular}

\section{RESEARCH RESULTS}

In all the sections of the studied drainage ditches intersecting forest phytocoenoses, the occurrence of 115 vascular plant species was found (Tab. 1), which represented 44 families and 81 genera (Tab. 2), whereas in the neighbouring phytocoenoses 69 species were recorded (Tab. 1), belonging to 29 families and 59 genera (Tab. 2). Both these habitats were characterised by the presence of only 46 common taxa.

In the flora of the studied drainage ditches, plants of the class Magnoliopsida were by far the dominant systematic group. Families of this class accounted for $68.2 \%$ of all families, genera $-70.4 \%$, and species $-61.7 \%$ (Tab. 2). The family Cyperaceae was represented in the greatest number - 15 species, as well as Poaceae -12 species and Rosaceae -9 species. However, most families were represented by single taxa (Tab. 1).

The flora within the phytocoenoses neighbouring the ditches has a similar systematic composition. The predominance of plants of the class Magnoliopsida was also noted here, as families of this class accounted for as much as $79.3 \%$ of all families, genera $-74.6 \%$, whereas species for $71.1 \%$ of the total flora (Tab. 2). The greatest number of species was noted from the family Poaceae -9 taxa. The families Rosaceae -7 taxa - and Cyperaceae - 6 taxa - were also represented in great numbers (Tab. 1).

The flora of the studied drainage ditches and neighbouring phytocoenoses represents nine plant lifeforms. Hemicryptophytes were decidedly the dominant group; 70 species were included in this category, i.e. $60.9 \%$ of the total ditch flora, and in the neighbouring phytocoenoses 35 taxa, which is $50.7 \%$ of their total flora. Hydrophytes, represented by 28 species $-24.3 \%$ of the flora, were an important group in the drainage ditches, second in terms of population size, whereas geophytes had a significant share in the neighbouring phytocoenoses; 12 species were included in them, accounting for $17.4 \%$ of the flora (Tab. 3).

In the flora of the studied drainage ditches and neighbouring phytocoenoses, a clear dominance of native species, which spontaneophytes are, was found. Native species accounted for $92.2 \%$ of the total ditch flora and $88.4 \%$ of the flora of the neighbouring phytocoenoses. At the same time, a characteristic feature of the flora of the investigated ecosystems is a clear predominance of non-synanthropic spontaneophytes. Only in the neighbouring phytocoenoses were 2 anthropophyte species noted, that is, alien species (Tab. 4), which included Consolida regalis Gray and Vicia tetrasperma (L.) Schreb.

Based on the division adopted by $\mathrm{Chmiel}$ (1993b), the flora of the studied drainage ditches was classified in 4 phytochoria, included in the boreal elements - Boreal Subkingdom (Euro-Siberian-BoreoAmerican). These are as follows:

- circumboreal subelement (CB) - Circumboreal Region;

- Euro-Siberian subelement (ES) - Euro-Siberian Region;

- Central European geographical group sOZ-E (E) - Central European Province;

- sub-Atlantic geographical group (sOZ) - Sub-Atlantic Province (Tab. 5).

Species whose range was outside the boundaries of the abovementioned phytochoria were included in 
overlapping elements. Cosmopolitan species (KOSM) are an element with the widest range, going beyond the boundaries of the Holarctic Kingdom.

In the flora of the studied drainage ditches, 65 species, accounting for $56.5 \%$ of the flora, belonged to a strictly defined phytochorion. Taxa of Euro-Siberian range were predominant among them - 39 species, i.e. $33.9 \%$ of the flora. Circumboreal and Central European elements, represented respectively by 15 species, i.e. $13 \%$, and 10 species, i.e. $8.7 \%$ of the flora, had a smaller proportion. Only one species, accounting for $0.9 \%$ of the flora, which was Carex elata All, represented sub-Atlantic range plants. Cosmopolitan species make up a large percentage of the flora. This group is represented by 18 taxa accounting for $15.7 \%$ of the ditch flora (Tab. 5).

In the flora of the neighbouring forest phytocoenoses, only 42 species belonged to a strictly defined phytochorion, which accounted for $60.9 \%$ of the flora. Taxa of Euro-Siberian range, represented by 20 species, i.e. $29 \%$ of the recorded flora, also dominated here. Compared to the ditch flora, cosmopolitan species, represented in the neighbouring phytocoenoses only by taxa accounting $8.7 \%$ of the species composition, showed a significantly smaller proportion (Tab. 5).

An analysis of the flora of the drainage ditches in terms of the continentality index showed that species neutral to continentality were by far dominant, since they accounted for as much as $97.4 \%$ of the flora of the drainage ditches, being represented by 112 taxa, and for $95.5 \%$ of the flora of the neighbouring phytocoenoses, which corresponds to 68 species of their flora. Both in the drainage ditches and in their neighbourhood, one sub-Atlantic species was recorded, found mainly in the western part of Poland, notably Genista pilosa. In the drainage ditches, the presence of one subcontinental taxon was found, occurring primarily in the eastern part of Poland Carex lasiocarpa (Tab. 1).

\section{DISCUSSION}

Research studies on the biodiversity of mezzoand microhabitats, which also include drainage ditches, are not sufficiently detailed, in particular that these habitats can be the place of occurrence of interesting biocenotic structures $(\mathrm{Hi} 11 \mathrm{bricht}-\mathrm{I} l \mathrm{kowska}$, 1998).

The species richness of the flora in the studied drainage ditches intersecting forest phytocoenoses, relative to the total flora of the Polesie National Park, is quite high. Species recorded in the study areas (115) were as much as $11.2 \%$ of the flora of the Polesie National Park. The species diversity of the flora of the forest phytocoenoses was almost half lower, and the number of species noted in the patches of these phytocoenoses was only 69 taxa, which accounted for $6.7 \%$ of the Park's total flora (Fijałkowski and I z d e b s k i , 2002).

In their floristic studies carried out in drainage ditches of a peat meadow area in Ko s zele w ki (Garb Lubawski - the Lubawa Hummock), Z a ł u s k i and K a m i ń s k a (1999) found that greater flora richness was noted in drainage ditches, both at their bottom and on slopes, than in neighbouring phytocoenoses. This is also confirmed by the study presented in this paper.

Proportions of life-form groups in the flora of a studied area depend directly on climatic factors, but they can also be affected by anthropopressure (Chmi e l, 1993a). An analysis of the life-form spectrum of the flora of the drainage ditches and their neighbourhood in the Polesie National Park showed that it is typical for this geographic zone. Hemicryptophytes were the dominant plant form in the ditches as well as in their neighbourhood. Due to greater moistening of the study areas, hydrophytes were an almost three time larger group - 28 taxa - in the drainage ditches than in their neighbourhood - 10 taxa.

One of the tasks set before research on flora is to explain the origin of taxa and to determine their status in autogenic and anthropogenic ecosystems, since the proportion, in terms of quality and quantity, of species of different historical-geographical groups can be a good indicator of the naturalness of the studied flora (C h m i e 1, 1993a). The analysis of the drainage ditch flora, in these terms, gave a quite clear, though not obvious picture that this special type of habitats, of only anthropogenic origin, was colonised by vegetation without the decisive human influence. Therefore, spontaneophytes - taxa which came to the study area or are native to it and can exist there without human participation - had the largest proportion in the ditch flora. This is indicated by the fact that as much as $92.2 \%$ of species recorded in the studied drainage ditches belong to the abovementioned group.

Similar correlations were observed in the flora of the phytocoenoses neighbouring the drainage ditches. Anthropophytes, that is, species which come from a given area or were introduced there and colonised the area only through human activity and persist thanks to that activity, were not found in the drainage ditches. Their presence was noted in the phytocoenoses neighbouring the ditches, but they occurred there in small numbers, since they accounted for as little as $2.9 \%$ of the total flora of the ditches' neighbourhood. Therefore, it can be stated that anthropogenic habitats of the drainage ditches were colonised by indigenous flora, characteristic for the study area, and man did not exert a strong pressure on their spe- 
cies composition. The lack of maintenance of the channels of the ditches and, in the first place, the establishment of the national park decided about the natural character of the flora of the drainage ditches in question.

The geographic distribution of species is primarily related to their tolerance to the variability of environmental factors in time and space $(\mathrm{Ch} \mathrm{mie} 1$, 1993a). Euro-Siberian and cosmopolitan species were the dominant range elements of the flora of the studied drainage ditches. Similar correlations were observed in the flora of the neighbouring phytocoenoses, though cosmopolitan species were by far a smaller group there.

\section{CONCLUSIONS}

1. In all the investigated sites of the drainage ditches, the occurrence of a total of 115 vascular plant species was found, which accounted for $11.2 \%$ of the Park's total flora. The drainage ditches are habitats important for the natural value of this area, and their richness and species diversity significantly enrich local biodiversity.

2. In the phytocoenoses neighbouring the drainage ditches, the occurrence of 69 vascular flora species was found, which accounted for $6.7 \%$ of the Park's flora. Hence, the species diversity of the drainage ditches was higher than that of the flora of the neighbouring habitats.

3. The analysis of the life-form spectrum of the flora of the drainage ditches in the Polesie National Park shows that it is typical for this geographic zone. Similar correlations were observed in the flora of the neighbouring phytocoenoses.

4. Spontaneophytes were the dominant historical-geographical group in the flora of the investigated watercourses. Anthropogenic habitats of the drainage ditches were colonised by native flora, characteristic for the study area, without significant human influence on their species composition.

\section{REFERENCES}

Chmiel J. 1993 a. Flora roślin naczyniowych wschodniej części Pojezierza Gnieźnieńskiego i jej antropogeniczne przeobrażenia w wieku XIX i XX. Część I. / Vascular plant flora of the eastern part of the Gniezno Lakeland and its anthropogenic transformations in the 19th and 20th centuries. Part I. Sorus, Poznań: 56-58, 97-98.

Chmiel J. 1993 b. Flora roślin naczyniowych wschodniej części Pojezierza Gnieźnieńskiego i jej antropogeniczne przeobrażenia w wieku XIX i XX. Część II. Atlas rozmieszczenia roślin. / Vascular plant flora of the eastern part of the Gniezno Lakeland and its anthropogenic transformations in the 19th and 20th centuries. Part II. Plant distribution atlas. Sorus, Poznań.
Ch mielew ski T. J. 1986. W sprawie utworzenia Parku Narodowego na Pojezierzu Łęczyńsko-Włodawskim. / On the establishment of a National Park in the WłodawaŁęczna Lakeland. Chrońmy Przyrodę Ojczystą, 42, (4): 61-67.

Chmielewski T. J., R a dwa n S. 1993. Zmiany stosunków ekologicznych w rejonie Poleskiego Parku Narodowego w ostatnich 75 latach. / / Changes in ecological relationships in the Polesie National Park area over the last 75 years. [In:] Radwan S., Karbowski Z., Sołtys M. (red.). Ekosystemy wodne i torfowiskowe w obszarach chronionych. Materiały konferencji „Funkcjonowanie ekosystemów wodnych i torfowiskowych w obszarach chronionych". / Aquatic and bog ecosystems in protected areas. Materials from the conference "The functioning of aquatic and bog ecosystems in protected areas". Krasne 28 - 29 June 1993 TWWP, PPN, Lublin: 13-25.

Chmielewski T. J., Sielewicz B. 1994. Ekologiczna waloryzacja terenu. / Ecological land evaluation. [In:] Radwan S. (red.). Środowisko przyrodnicze w strefie oddziaływania Kanału Wieprz-Krzna. / Natural environment in the impact area of the Wieprz-Krzna Canal. TWWP, Lublin: 9-28.

Fijałkowski D. 1959. Wykaz rzadszych roślin Lubelszczyzny. Część III. / List of more rare species of the Lublin region. Part II. Fragm. Florist. Geobot. Ann. V, Pars 1: 11-35.

Fijałkowski D., Izdebski K. 2002. Flora Poleskiego Parku Narodowego. Rośliny naczyniowe. / Flora of the Polesie National Park. Vascular plants. [In:] Radwan S. (red.). Poleski Park Narodowy. Monografia przyrodnicza. / The Polesie National Park. Natural monograph. Wyd. MORPOL, Lublin: 103-114.

Hillbricht-Ilkowska A. 1998. Różnorodność biologiczna siedlisk słodkowodnych - problemy, potrzeby, działania. / Biological diversity of freshwater habitats - problems, needs, actions. [In:] Kraska M. (red.). Bioróżnorodność w środowisku wodnym. / Biodiversity in the aquatic environment. Idee Ekologiczne, Tom 13, Ser. Szkice. Wyd. Sorus, Poznań, 17: 13-54.

Kałamucki K., Cebrykow P., Grzechnik L. 2001. Poleski Park Narodowy. Mapa turystyczna 1:50000. Kartpol s.c., Lublin.

Michalczyk Z., Turczyński M. 1998. Przekształcenia i zagrożenia hydrosfery. / Transformations of and threats to the hydrosphere. [In:] Harasimiuk M., Michalczyk., Turczyński M. (red.), Jeziora łęczyńsko-włodawskie. Monografia przyrodnicza. / Lakes of the Łęczna and Włodawa area. Natural monograph. Biblioteka Monitoringu Środowiska, Lublin: 157-164.

Turczyński M., Michalczyk Z., Bartoszewski S. 2000. Geograficzne aspekty ochrony i użytkowania hydrosfery Pojezierza Łęczyńsko-Włodawskiego. / Geographical aspects of protection and use of the hydrosphere of the Łęczna-Włodawa Lakeland. [In:] Radwan S., Lorkiewicz Z. (red.). Problemy ochrony i użytkowania obszarów wiejskich o dużych walorach przy- 
rodniczych. / Problems of protection and use of rural areas with great natural values. Wydawnictwo UMCS, Lublin: 57-61.

Wilgat T. 1991. Stosunki wodne Pojezierza ŁęczyńskoWłodawskiego. Zmiany stosunków wodnych pod wpływem gospodarki. / Water relations of the Łęczyńsko-Włodawskie Lakeland. Economy-driven changes in water relations. [In:] Kleczkowski A. S. (ed.). Studia Ośrodka Dokumentacji Fizjograficznej. I Studies of the Physiographic Documentation Centre. PAN, Wrocław, Warszawa, Kraków, 19: 48-52.

Załuski T., Kamińska A. 1999. Rola rowów melioracyjnych jako refugiów flory torfowiskowej na przykładzie kompleksu łąk w Koszelewkach. / The role of drainage ditches as refuges for bog flora on the example of a meadow complex in Koszelewki. Folia Universitatis Agriculturae Stetinensis, Agricultura, 197 (75): 373-376.

Zarzycki K., Trzcińska-Tacik H., Różański W., Szeląg Z., Wołek J., Korzeniak U. 2002. Ecological indicator values of vascular plants of Poland. Ekologiczne liczby wskaźnikowe roślin naczyniowych Polski. W. Szafer Institute of Botany, Polish Academy of Science, Kraków.

\section{Flora naczyniowa rowów melioracyjnych na terenach leśnych Poleskiego Parku Narodowego}

\section{Streszczenie}

Praca przedstawia wyniki badań florystycznych prowadzonych w latach 2003-2005 na terenie Poleskiego Parku Narodowego. Celem niniejszej pracy było porównanie flory inicjalnych stadiów sukcesyjnych w rowach melioracyjnych, z florą przekształcających się wskutek odwodnienia, sąsiadujących terenów leśnymi. Na czterech wyznaczonych powierzchniach badań, a także w fitocenozach leśnych sporządzono spisy florystyczne. Wyniki badań terenowych poddano analizie kameralnej pod kątem przynależności systematycznej flory, udziału form życiowych roślin, a także grup historyczno-geograficznych i grup zasięgowych.

W składzie gatunkowym, badanych rowów melioracyjnych dominującą grupą form życiowych roślin wyższych są hemikryptofity, podczas gdy w zbiorowiskach sąsiadujących z nimi florę reprezentują zarówno hemikryptofity jak i megafanerofity.

Pod względem historyczno-geograficznym flora rowów melioracyjnych, jak i zbiorowisk sąsiadujących, reprezentuje zdecydowanie grupę spontaneofitów. Antropogeniczne siedliska, jakie wykształciły się w obrębie rowów melioracyjnych zasiedlone zostały przez gatunki flory rodzimej charakterystyczne dla terenu Poleskiego Parku Narodowego. 